\title{
Salivary Amylase Induction by Tannin-Enriched Diets as a Possible Countermeasure Against Tannins
}

\author{
G. da Costa • E. Lamy • F. Capela e Silva • J. Andersen • \\ E. Sales Baptista $\cdot$ A. V. Coelho
}

Received: 24 July 2007/ Revised: 26 November 2007/ Accepted: 28 November 2007 / / Published online: 6 February 2008

(C) Springer Science + Business Media, LLC 2007

\begin{abstract}
Tannins are characterized by protein-binding affinity. They have astringent/bitter properties that act as deterrents, affecting diet selection. Two groups of salivary proteins, proline-rich proteins and histatins, are effective precipitators of tannin, decreasing levels of available tannins. The possibility of other salivary proteins having a co-adjuvant role on host defense mechanisms against
\end{abstract}

G. da Costa and E. Lamy have contributed equally to this work.

G. da Costa $\cdot$ A. V. Coelho $(\triangle)$

ITQB-Instituto de Tecnologia Química e Biológica,

Oeiras, Portugal

e-mail: varela@itqb.unl.pt

E. Lamy $\cdot$ E. Sales Baptista

ICAM-Instituto de Ciências Agrárias Mediterrânicas,

Universidade de Évora,

Évora, Portugal

E. Lamy $\cdot$ E. Sales Baptista

Departamento de Zootecnia, Universidade de Évora,

Évora, Portugal

F. Capela e Silva

Departamento de Biologia, Universidade de Évora,

Évora, Portugal

F. Capela e Silva

Centro de Investigação em Ciências e Tecnologias da Saúde,

Universidade de Évora,

Évora, Portugal

\section{J. Andersen}

Department of Biochemistry and Molecular Biology, University

of Southern Denmark,

Odense, Denmark

\section{A. V. Coelho}

Departamernto de Química Universidade de Évora,

Évora, Portugal tannins is unknown. In this work, we characterized and compared the protein profile of mice whole saliva from animals fed on three experimental diets: tannin-free diet, diet with the incorporation of $5 \%$ hydrolyzable tannins (tannic acid), or diet with 5\% condensed tannins (quebracho). Protein analysis was performed by one-dimensional gel electrophoresis combined with Matrix-Assisted Laser Desorption Ionization-Time of Flight mass spectrometry to allow the dynamic study of interactions between diet and saliva. Since abundant salivary proteins obscure the purification and identification of medium and low expressed salivary proteins, we used centrifugation to obtain saliva samples free from proteins that precipitate after tannin binding. Data from Peptide Mass Fingerprinting allowed us to identify ten different proteins, some of them showing more than one isoform. Tannin-enriched diets were observed to change the salivary protein profile. One isoform of $\alpha$-amylase was overexpressed with both types of tannins. Aldehyde reductase was only identified in saliva of the quebracho group. Additionally, a hypertrophy of parotid salivary gland acini was observed by histology, along with a decrease in body mass in the first 4 days of the experimental period.

Keywords Salivary proteins $\cdot$ Amylase $\cdot$ SDS-PAGE $\cdot$ Mass spectrometry Defense mechanisms against tannins $\cdot$ Taste

\section{Introduction}

Tannins are plant secondary metabolites (PSMs) with an enormous structural diversity and are found worldwide in many families of higher plants. One of the characteristic properties of these natural high-molecular-mass polyphenols is their high capacity to bind proteins, forming 